\title{
Present status and phytosociology of Critically Endangered Streptocaulon sylvestre Wight (Apocynaceae) in its only natural habitat in North Bengal University campus, India
}

\author{
Priyanka Das, Chandra Ghosh ${ }^{2}$ and A. P. Das ${ }^{1}$ \\ Department of Tea Science, University of North Bengal, Darjeeling - 734013, West Bengal, India \\ ${ }^{1}$ Department of Botany, Rajiv Gandhi University, Rono Hills, Doimukh 791112, Arunachal Pradesh, India \\ (Formerly of: University of North Bengal) \\ ${ }^{2}$ Communicating author, E-mail: amichandra07@gmail.com
}

[Received 26.04.2020; Revised 17.06.2020; Accepted 27.06.2020; Published 30.06.2020]

\begin{abstract}
After the re-discovery of Streptocaulon sylvestre Wight (Apocynaceae) interest grew for its conservation. The species has been declared as Critically Endangered (CR) by the Ministry of Environment \& Forest, Govt. of India though attempt to its in situ conservation remained limited to the creation a 'Streptocaulon sylvestre Reserve' (SSR) within the campus of North Bengal University on $31^{\text {st }}$ December 2013. Previous attempts for its ex situ propagation, expansion of the area of occurrence and its domestication as a garden plant also were not positive. So it appears that the species should be conserved in in situ condition only under strict regular monitoring.

The present attempt of understanding the vegetation structure of the SSR was undertaken during a period when the seasons were not favourable for the better growth of most of the plants. Even then a rich diversity of flora of the reserve has been elucidated. The most dominant species in the reserve is Cymbopogon pendulus and some other associated species.
\end{abstract}

The present status of the species and the probable strategies for its maintenance-cumconservation in its only known habitat has been discussed in this paper.

Key words: Streptocaulon sylvestre, Critically Endangered, Phytosociology, In situ Conservation

\section{INTRODUCTION}

The richness of the floristic diversity of Terai and Duars regions of West Bengal is well-known (Das 1995, 2013; Das et al. 2010). Large number of RET and endemic species are known to grow in this area and to conserve the biological diversity of this region quite a good number of Protected Areas (PA) are demarcated there and those are under operation (Das \& Bhujel 2008; Sen \& Mandal 2018; Das \& Lungphi 2019). No doubt, these PAs are housing many important RET, endemic and economically exploitable species. But, at the same time, there are some such endangered species those are growing outside the PAs and needs immediate care and attention for their conservation.

Streptocaulon sylvestre Wight (Apocynaceae) is a small suffrutescent, strictly procumbent herb with milky latex that never gets rooted from branches. Originally collected by Fr. Buchanan Hamilton (Ham. Herb. No. 763; CAL) from the Sannyasikata village (Wight 1834; Das 1996) in the district of Jalpaiguri (West Bengal, India) on $7^{\text {th }}$ of April 1809, the plant was doubtfully recognized by him as a species of Periploca. Later, in 1834 Robert Wight named it as Streptocaulon sylvestre (Wight 1834).

Streptocaulon sylvestre is one extremely rare plant and is reported to grow only in the foothill regions of Eastern Himalaya, namely Siliguri (Siliguree) and Sikkim Himalayas (Hooker 
1883; Prain 1903; Watson 1999). The species has been declared as Critically Endangered (CR) by MoEF, Govt. of India, through the Gazette Notification no. S.O.769(E)-In exercise of the powers conferred by section 38 of the biological diversity Act, 2002 ( 18 of 2003), on $31^{\text {st }}$ March 2010, by the Government of India (Figure 1; serial no. 2).

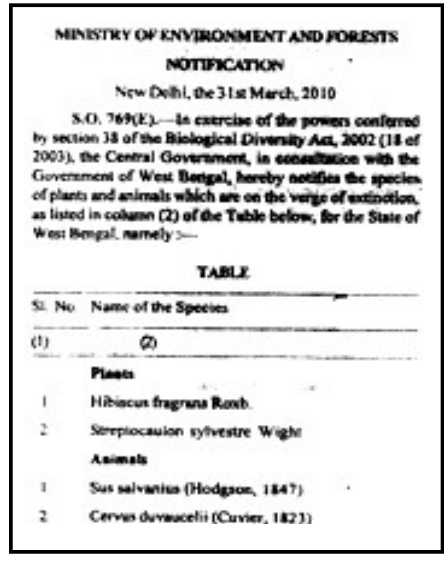

Figure 1. MoEF, Govt. of India, through the Gazette Notification no. S.O.769(E).

With initiative of the senior author (APD), the North Bengal University authority has fenced one Streptocaulon rich area of about $7500 \mathrm{sq} \mathrm{m}$ for its in situ conservation in 2013. The area was then declared as the 'Streptocaulon sylvestre Reserve' (SSR) and the species was provided with a vernacular name 'Uttara' by the then Honb'le Vice-Chancellor Prof. Samir Kumar Das.

Phytosociology is the branch of science that deals with the plant communities, their composition and development, and the relationships between different species living together. This system is also used for classifying different plant communities (Paczoski 1896; Braun-Blanquet 1932; Poore 1955). In SSR the species is sharing the habitat with many other species including its preferred associates like Borreria alata, Cymbopogon pendulus, Imperata cylindrica, Saccharum spontaneum, etc. (Kadir 2001; Kadir \& Das 2002; Kadir et al. 2009).

The species needs declaration as 'Pride of NBU' as suggested by the then Secretary General of Botanic Gardens

Conservation International in a personal communication to APD.

\section{Why Endangered?}

The main cause for a species to become endangered is the loss and disturbances of its natural habitat, mainly due to the expansion of human settlements and its related activities. In addition, there are some natural disasters like flood, forest-fire, over-powered by associated species, loss of preferred association, diseases and pest infestation, grazing of animals, etc. can also render a species endangered.

Streptocaulon sylvestre was never had a wide distribution and was endemic to Terai-Duars region of Bengal and Bihar (Das 1996), a region, that was covered with almost undisturbed vegetation even just 100 years earlier. Uncontrolled expansion of human settlements, tea garden, broad surface communication systems, etc. led to the extensive loss of natural habitat for the wildlife, both plants and animals in this entire area. The process has also affected the survival of S. sylvestre restricting it to about three acres area of the land inside the campus of the University of North Bengal. Due to different developmental activities of the University, it is now reduced to a small patch that is now SSR and another very small patch behind the Zoology Department building. However, physical searches since long in its previously known habitat, including the Type-locality at Snnyasikata failed to relocate its presence.

The experiments on the reproductive capacity of $S$. sylvestre shows that the rate of seedgermination is quite high but the rate of seedling survival is extremely poor and the microhabitat preference is very strict (Kadir \& Das 2007).

However, its population and growth inside the SSR is quite good. The present work was undertaken to understand the species richness of the SSR to characterise the vegetation type and to assess the present status of this Critically Endangered and endemic species of India. Also the generated data will be helpful to develop necessary strategies for its proper conservation. 


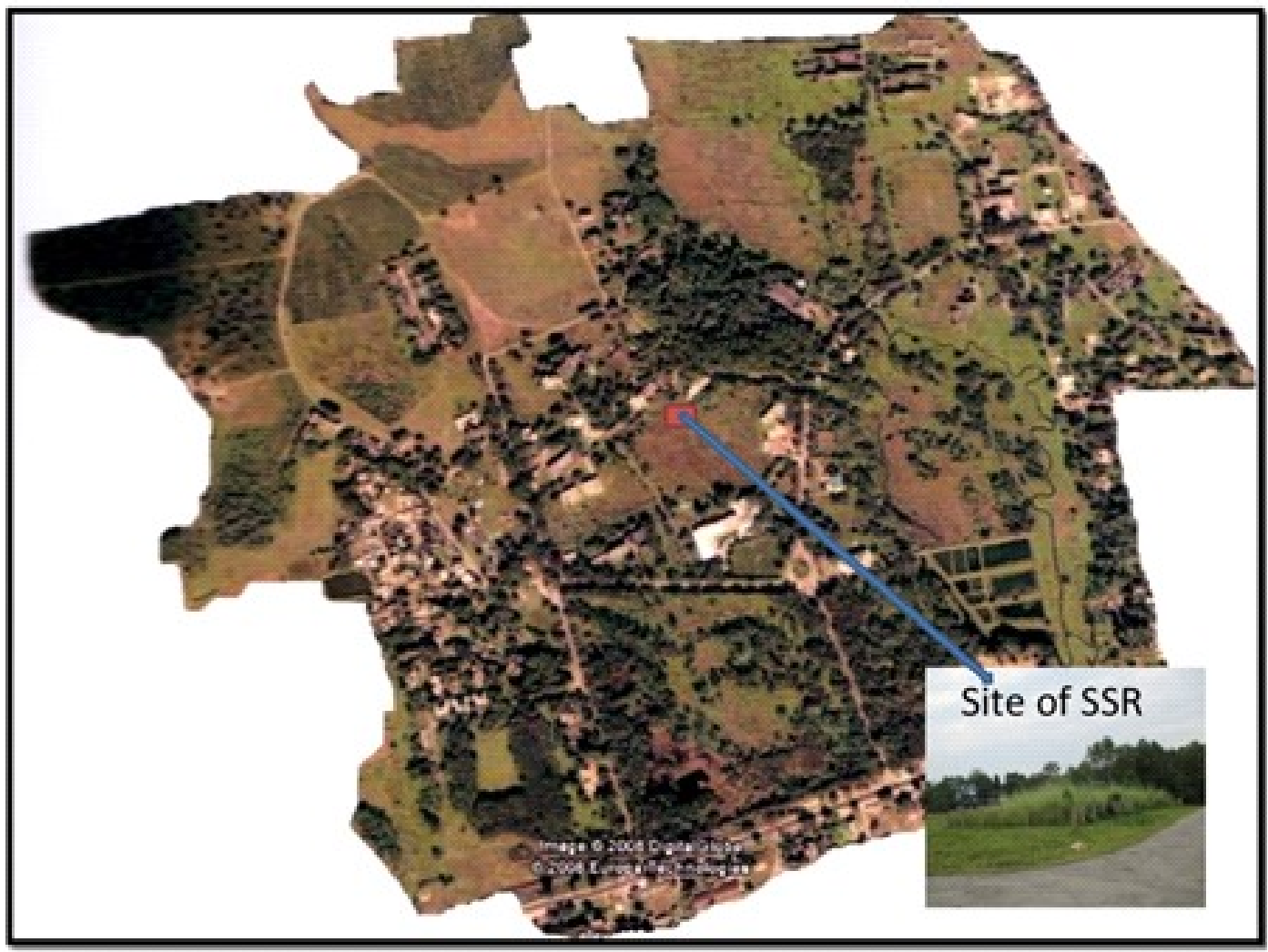

Figure 2. Arial photograph of North Bengal University campus (source: http//www.nbu.co.org)

\section{Study area}

\section{METHODOLOGY}

'Streptocaulon sylvestre Reserve' (SSR), which was established on the last day of the year 2013 (Anonymous 2014) and is located at the central sector of North Bengal University campus [26 $22^{\circ} 20^{\prime \prime}$ to $26^{\circ} 43^{\prime} 20^{\prime \prime} \mathrm{N}$ and $88^{\circ} 20^{\prime} 55^{\prime \prime}$ to $88^{\circ} 21^{\prime} 31^{\prime \prime} \mathrm{E}$ ] with an average altitude of c. $130 \mathrm{~m}$ AMSL (Figure 2). The SSR area is situated on upland (terrestrial) and is now bounded by barbed wire fencing (beside Geology Department).

The drainage is good and never face water-logging. The maximum summer temperature varies between $35^{\circ} \mathrm{C}$ to $37^{\circ} \mathrm{C}$ and minimum winter temperature is c. $7^{\circ} \mathrm{C}$ with average maximum and minimum atmospheric humidity $93.09 \%$ and $57.14 \%$ respectively. The annual average rainfall at the campus is $348.51 \mathrm{~cm}$ (Sharma 2007; Kadir et al. 2009). Previously the habitat-area was covered with the dense population of Cymbopogon pendulus and was suffering from fire almost every year.

According to Champion \& Seth's (1968) classification of Indian Forest Types, the NBU Campus area was covered with mixed deciduous type of forest, dominated mainly by Saal (Shorea robusta). But, now, except a very small patch of Saal forest, located at around $80 \mathrm{~m}$ away from SSR, no other area is covered with natural forested vegetation inside or on surroundings of the campus.

\section{Phytosociological Observation}

The entire area of SSR has been surveyed phytosociologically using quadrate technique. As the habitat is a grassland, the standard quadrate size of 1 x $1 \mathrm{~m}$ has been employed for basic list- 

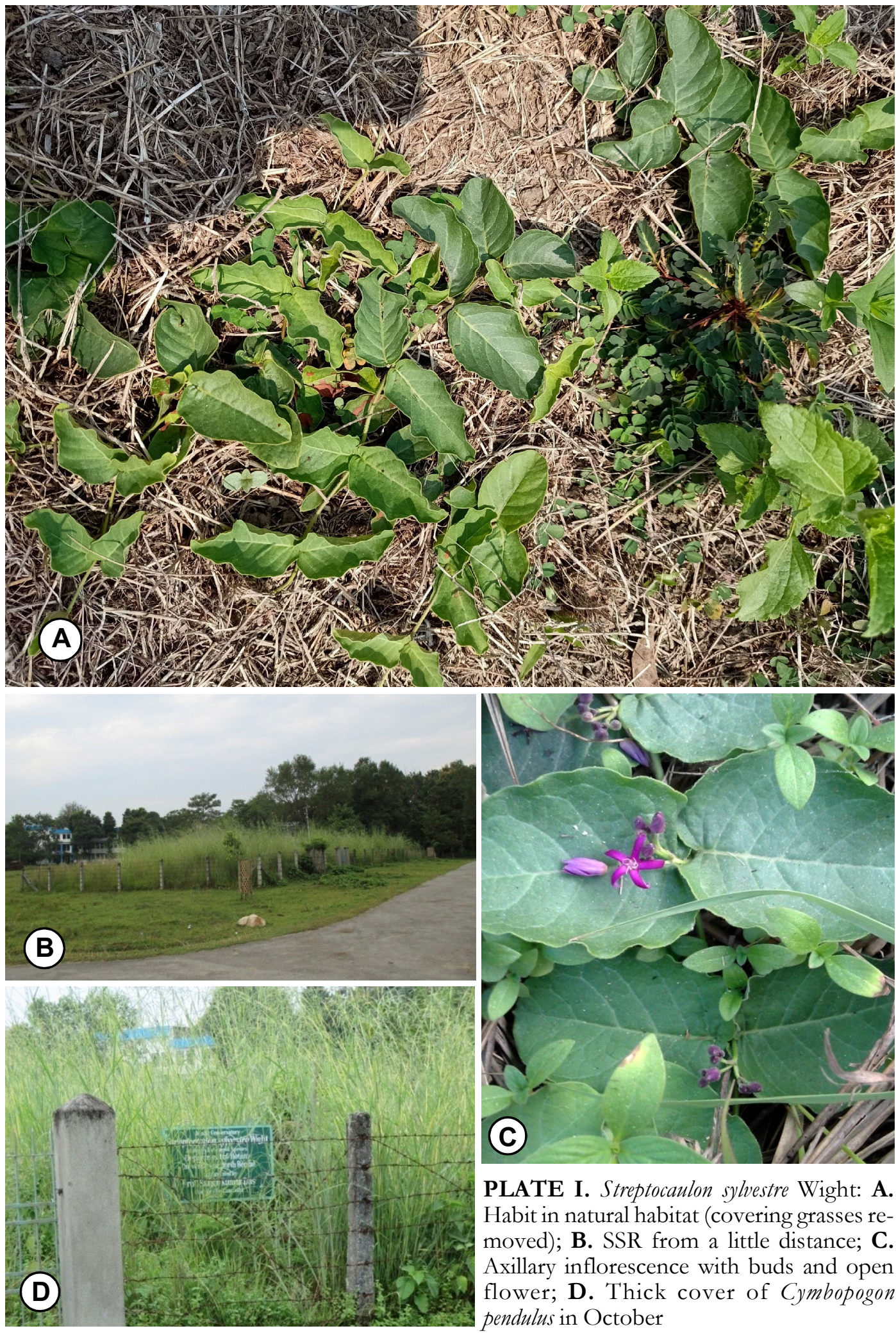

PLATE I. Streptocaulon sylvestre Wight: A. Habit in natural habitat (covering grasses removed); B. SSR from a little distance; C. Axillary inflorescence with buds and open flower; D. Thick cover of Cymbopogon pendulus in October 
count data. Sampling was done by semi-random quadrates on three equidistant line-transects. In the present study, a total of 100 quadrates have been studied for three seasons: Spring (February to March), Summer (April to May) and Monsoon (June to July) for the years 2016 and 2019 to achieve fair idea about the vegetation and its composition.

This list-count data is then processed to determine the second level data like RD, RF, RA and IVI (Mirsa (1966), Shimwell (1971), Tripathi \& Mishra (1971), Phillp (1959), Das \& Lahiri (1997), Kadir (2001) and Rai (2006)).

The species diversity and Species Richness of SSR were determined using the ShannonWeiner Index (1963) and Menhinick's Index (1964). Also, the Concentration of Dominance of species and Similarity Index were determined by Simpson's Index (1949) and Sorensen's Index of Similarity (1968) respectively.

\section{Shannon-Weiner Index (SDI):}

Species Diversity Index [Shannon-Weiner Index] or SDI was calculated using the formula:

$$
\mathrm{H}^{\prime}=- \text { Ó }[(\mathrm{ni} / \mathrm{N}) \ln (\mathrm{ni} / \mathrm{N})]
$$

[Where, ' $\mathrm{H}$ ' is index value; 'ni' number of individuals of a species; and ' $\mathrm{N}$ ' total number of species in the habitat]

Species Richness [Menhinick's Index (1964)] with the formula:

$$
\mathrm{D}=\mathrm{S} /{ }^{\prime} \mathrm{N}
$$

[Where, ' $\mathrm{D}$ ' is the index value; $\mathrm{S}$ ' total number of species; ' $\mathrm{N}$ ' total number of individuals of all species]

Concentration of Dominance [Simpson's index, 1949] measured with following formula:

$$
\ddot{\mathrm{e}}=\mathrm{O}_{\mathrm{pi}}{ }^{2}
$$

[where, ' $\ddot{e}$ ' is the index value; 'pi' is the proportional abundance of the $i^{\text {th }}$ species

$[\mathrm{Pi}=$ ni/N ['ni' number of individuals of a species; ' $\mathrm{N}$ ' total number of species in the habitat type]

Margalef indices ( $\mathbf{R}_{\mathbf{1}}$ ) (Margalef 1968):

$$
\mathrm{R}_{\mathrm{I}}=\mathrm{S}-1 / \text { In }(\mathrm{n})
$$

[Where, $\mathrm{S}=$ Total number of species; nnumber of individuals of a species]

The voucher specimens were processed into mounted herbarium-sheets following Jain and Rao $(1977 / 2016)$ and the identified using floras covering this region including Prain (1903); Hara (1966, 1971); Hara et al. (1978, 1979, 1982); Grierson \& Long (1983 - 2001); Noltie (1994, 2000); Anonymous (1997, 2015, 2016) and by matching at the NBU-Herbarium. For up-dated nomenclature and for family delimitation www.plantsoftheworldonline.org has been consulted. These specimens will be deposited in the Herbarium of the Tea-Science Department of North Bengal University after the works are over.

\section{RESULT}

The result of phytosociological survey through quadrate sampling in the 'Streptocaulon sylvestre Reserve' (SSR) inside the North Bengal University campus has been presented in Tables $1-3$ for different study seasons. A total of 100 quadrates have been studied for three seasons to get fair idea about the vegetation composition. 
62 Phytosociology of 'Streptocaulon sylvestre Reserve'

Spring time vegetation: For the spring flora the vegetation was sampled during February March and has recorded 28 species of plants. Analysis of the collected data has been presented in Table 1.

Table 1. Analysis of spring-season (February - March) data [Abbreviations used: $\mathbf{R A}=$ Relative Abundance; $\mathbf{R D}=$ Relative Density; $\mathbf{R F}=$ Relative Frequency; IVI = Important Value Index. Habitgroups: $\mathbf{C}=$ Climber; $\mathbf{H}=$ Herb; $\mathbf{S}=$ Shrub; $\mathbf{S H}=$ Suffrutescent Herb; $\mathbf{S S}=$ Suffrutescent Shrub; $\mathbf{T}=$ Tree]

\begin{tabular}{|c|c|c|c|c|c|}
\hline TAXA & Habit & RA & $\mathbf{R D}$ & $\mathbf{R F}$ & IVI \\
\hline Ageratum conyzoides L. [Compositae] & $\mathrm{H}$ & 3.61 & 3.46 & 6.14 & 13.20 \\
\hline $\begin{array}{l}\text { Amaranthus blitum subsp. oleraceus (L.) Costea } \\
\text { [Amaranthaceae] }\end{array}$ & $\mathrm{H}$ & 3.21 & 1.15 & 2.30 & 6.66 \\
\hline Clerodendrum infortunatum L. [Labiatae] & SS & 4.27 & 8.36 & 12.53 & 25.16 \\
\hline Cyanthillium cinereum (L.) H.Rob. [Compositae] & $\mathrm{H}$ & 1.80 & 0.29 & 1.02 & 3.12 \\
\hline $\begin{array}{l}\text { Cymbopogon pendulus (Nees ex Steud.) W.Watson } \\
\text { [Gramineae] }\end{array}$ & $\mathrm{SH}$ & 13.17 & 42.07 & 20.46 & 75.70 \\
\hline Crotalaria pallida Aiton [Leguminosae] & $\mathrm{H}$ & 1.44 & 0.06 & 0.26 & 1.76 \\
\hline $\begin{array}{l}\text { Chromolaena odorata (L.) R.M.King \& H.Rob. } \\
\text { [Compositae] }\end{array}$ & SS & 4.44 & 2.31 & 3.32 & 10.07 \\
\hline Desmodium triflorum (L.) DC. [Leguminosae] & $\mathrm{H}$ & 3.89 & 2.02 & 3.32 & 9.23 \\
\hline $\begin{array}{l}\text { Flueggea virosa (Roxb. ex Willd.) Royle } \\
\text { [Euphorbiaceae] }\end{array}$ & $\mathrm{S}$ & 3.37 & 0.40 & 0.77 & 4.54 \\
\hline Ipomoea linearifolia Hook. f. [Convolvulaceae] & $\mathrm{C}$ & 1.44 & 0.06 & 0.26 & 1.76 \\
\hline Imperata cylindrica (L.) Raeusch. [Gramineae] & $\mathrm{H}$ & 1.44 & 0.06 & 0.26 & 1.76 \\
\hline Ichnocarpus frutescens (L.) W.T.Aiton [Apocynaceae] & $\mathrm{C}$ & 2.41 & 0.29 & 0.77 & 3.46 \\
\hline Leucas zeylanica (L.) W.T.Aiton [Labiatae] & $\mathrm{H}$ & 2.89 & 0.23 & 0.51 & 3.63 \\
\hline Mikania micrantha Kunth [Compositae] & $\mathrm{C}$ & 2.89 & 0.23 & 0.51 & 3.63 \\
\hline Mitracarpus hirtus (L.) DC. [Rubiaceae] & $\mathrm{H}$ & 3.05 & 1.09 & 2.30 & 6.44 \\
\hline Mimosa pudica L. [Leguminosae] & $\overline{\mathrm{H}}$ & 3.48 & 2.36 & 4.35 & 10.19 \\
\hline Pueraria phaseoloides (Roxb.) Benth. [Leguminosae] & $\mathrm{C}$ & 3.66 & 1.90 & 3.32 & 8.89 \\
\hline Premna mollissima Roth [Labiatae] & $\mathrm{T}$ & 3.25 & 0.52 & 1.02 & 4.79 \\
\hline Pennisetum glaucum (L.) R.Br. [Gramineae] & $\mathrm{H}$ & 1.44 & 0.06 & 0.26 & 1.76 \\
\hline Rungia pectinata (L.) Nees [Acanthaceae] & $\mathrm{H}$ & 9.34 & 13.43 & 9.21 & 31.98 \\
\hline Streptocaulon sylvestre Wight [Apocynaceae] & $\mathrm{SH}$ & 4.26 & 6.80 & 10.23 & 21.29 \\
\hline Sporobolus fertilis (Steud.) Clayton [Gramineae] & $\mathrm{H}$ & 5.91 & 7.32 & 7.93 & 21.16 \\
\hline Senna tora (L.) Roxb. [Leguminosae] & $\mathrm{H}$ & 1.44 & 0.12 & 0.51 & 2.07 \\
\hline Triumfetta rhomboidea Jacq. [Malvaceae] & $\mathrm{H}$ & 4.49 & 4.84 & 6.91 & 16.24 \\
\hline Toona ciliata M.Roem. [Meliaceae] & $\mathrm{T}$ & 1.44 & 0.06 & 0.26 & 1.76 \\
\hline Tridax procumbens (L.) L. [Compositae] & $\mathrm{H}$ & 2.89 & 0.12 & 0.26 & 3.26 \\
\hline Trema orientale (L.) Blume [Cannabaceae] & $\mathrm{T}$ & 2.89 & 0.23 & 0.51 & 3.63 \\
\hline Zanonia indica L. [Cucurbitaceae] & $\mathrm{C}$ & 2.17 & 0.17 & 0.51 & 2.85 \\
\hline
\end{tabular}


Different indices calculated for the Spring-time vegetation are:

Simpsons Index: $1=0.44$

Menhinick Indices: $\mathrm{D}=0.67$

Margalef Indices: R1 $=1.80$

Summer time vegetation: The quadrate sampling for summer data was conducted during April - May and has recorded 35 species. Analysis of the collected data has been presented in Table 2.

Table 2. Analysis of spring-season (April - May) data

[Abbreviations used: as for Table- 1]

\begin{tabular}{|c|c|c|c|c|c|}
\hline TAXA & Habit & RA & RD & RF & IVI \\
\hline Amaranthus spinosus L. [Amaranthaceae] & $\mathrm{H}$ & 0.60 & 0.42 & 4.15 & 5.16 \\
\hline Argyreia nervosa (Burm.f.) Bojer [Convolvulaceae] & $\mathrm{C}$ & 0.71 & 0.06 & 0.52 & 1.29 \\
\hline Ageratum conyzoides L. [Compositae] & $\mathrm{H}$ & 1.00 & 0.79 & 4.66 & 6.46 \\
\hline Bidens pilosa L. [Compositae] & $\mathrm{H}$ & 0.44 & 0.08 & 1.04 & 1.56 \\
\hline Clerodendrum infortunatum L. [Labiatae] & SS & 0.39 & 0.17 & 2.59 & 3.15 \\
\hline Crotalaria pallida Aiton [Leguminosae] & $\mathrm{H}$ & 0.63 & 0.39 & 3.63 & 4.65 \\
\hline Cyanthillium cinereum (L.) H.Rob. [Compositae] & $\mathrm{H}$ & 0.18 & 0.02 & 0.52 & 0.71 \\
\hline $\begin{array}{l}\text { Cymbopogon pendulus (Nees ex Steud.) W.Watson } \\
\text { [Gramineae] }\end{array}$ & $\mathrm{SH}$ & 1.37 & 2.41 & 10.36 & 14.15 \\
\hline $\begin{array}{l}\text { Chromolaena odorata (L.) R.M.King \& H.Rob. } \\
\text { [Compositae] }\end{array}$ & SS & 28.87 & 27.94 & 5.70 & 62.51 \\
\hline Desmodium triflorum (L.) DC. [Leguminosae] & $\mathrm{H}$ & 2.38 & 1.46 & 3.63 & 7.47 \\
\hline Flueggea virosa (Roxb. ex Willd.) Royle [Phyllanthaceae] & $\mathrm{S}$ & 0.44 & 0.08 & 1.04 & 1.56 \\
\hline Hyptis suaveolens (L.) Poit. [Labiatae] & $\mathrm{H}$ & 17.55 & 15.44 & 5.18 & 38.17 \\
\hline Imperata cylindrica (L.) Raeusch. [Gramineae] & $\mathrm{H}$ & 0.71 & 0.12 & 1.04 & 1.87 \\
\hline Ichnocarpus frutescens (L.) W.T.Aiton [Apocynaceae] & $\mathrm{C}$ & 0.27 & 0.09 & 2.07 & 2.43 \\
\hline Ipomoea linearifolia Hook.f. [Convolvulaceae] & $\mathrm{C}$ & 0.35 & 0.03 & 0.52 & 0.90 \\
\hline Ipomoea quamoclit L. [Convolvulaceae] & $\mathrm{C}$ & 0.71 & 0.12 & 1.04 & 1.87 \\
\hline Leucas zeylanica (L.) W.T.Aiton [Labiatae] & $\mathrm{H}$ & 0.18 & 0.02 & 0.52 & 0.71 \\
\hline Litsea lancifolia (Roxb.ex Nees) Fern.-Vill. [Lauraceae] & $\mathrm{T}$ & 0.35 & 0.03 & 0.52 & 0.90 \\
\hline Mikania micrantha Kunth [Compositae] & $\mathrm{C}$ & 0.41 & 0.11 & 1.55 & 2.08 \\
\hline Mitracarpus hirtus (L.) DC. [Rubiaceae] & $\mathrm{H}$ & 30.23 & 39.90 & 7.77 & 77.90 \\
\hline Mimosa pudica L. [Leguminosae] & $\mathrm{H}$ & 0.53 & 0.19 & 2.07 & 2.79 \\
\hline Pueraria phaseoloides (Roxb.) Benth. [Leguminosae] & $\mathrm{C}$ & 0.69 & 0.79 & 6.74 & 8.22 \\
\hline Premna mollissima Roth [Labiatae] & $\mathrm{T}$ & 0.39 & 0.17 & 2.59 & 3.15 \\
\hline Pennisetum glaucum (L.) R.Br. [Gramineae] & $\mathrm{H}$ & 0.50 & 0.26 & 3.11 & 3.87 \\
\hline Rungia pectinata L. [Acanthaceae] & $\mathrm{H}$ & 0.26 & 0.20 & 4.66 & 5.12 \\
\hline Streptocaulon sylvestre Wight [Apocynaceae] & $\mathrm{SH}$ & 0.40 & 0.28 & 4.15 & 4.82 \\
\hline Sporobolus fertilis (Steud.) Clayton [Gramineae] & $\mathrm{H}$ & 4.89 & 6.88 & 8.29 & 20.06 \\
\hline Senna tora (L.) Roxb. [Leguminosae] & $\mathrm{H}$ & 0.77 & 0.20 & 1.55 & 2.52 \\
\hline Senna occidentalis (L.) Link [Leguminosae] & $\mathrm{H}$ & 0.65 & 0.17 & 1.55 & 2.37 \\
\hline Trema orientalis (L.) Blume [Cannabaceae] & $\mathrm{T}$ & 0.35 & 0.03 & 0.52 & 0.90 \\
\hline Triumfetta rhomboidea Jacq. [Malvaceae] & $\mathrm{H}$ & 1.57 & 0.97 & 3.63 & 6.16 \\
\hline Toona ciliata M.Roem. [Meliaceae] & $\mathrm{T}$ & 0.35 & 0.03 & 0.52 & 0.90 \\
\hline Tridax procumbens L. [Compositae] & $\mathrm{H}$ & 0.27 & 0.05 & 1.04 & 1.35 \\
\hline Wrightia tinctoria R.Br. [Apocynaceae] & $\mathrm{T}$ & 0.35 & 0.03 & 0.52 & 0.90 \\
\hline Zanonia indica L. [Cucurbitaceae] & $\mathrm{C}$ & 0.27 & 0.05 & 1.04 & 1.35 \\
\hline
\end{tabular}


64 Phytosociology of 'Streptocaulon sylvestre Reserve'

Different indices calculated for the Summer-time vegetation are:

Simpsons Index: $1=0.23$

Menhinick Indices: $\mathrm{D}=0.44$

Margalef Indices: R1 $=0.82$

The monsoon data: The third round survey for the monsoon (June - July) vegetation recorded only 31 species and has been presented in Table 3 .

Table: 3. Analysis of Rainy-season (June - July) data

[Abbreviations used: as in Table - 1]

\begin{tabular}{|c|c|c|c|c|c|}
\hline TAXA & Habit & RA & RD & $\mathbf{R F}$ & IVI \\
\hline Ageratum conyzoides L. [Compositae] & $\mathrm{H}$ & 1.49 & 0.16 & 0.67 & 2.32 \\
\hline Amaranthus spinosus L. [Amaranthaceae] & $\mathrm{H}$ & 1.19 & 0.21 & 1.12 & 2.53 \\
\hline Argyreia nervosa (Burm.f.) Bojer [Convolvulaceae] & $\mathrm{C}$ & 0.10 & 0.04 & 0.22 & 1.25 \\
\hline Bidens pilosa L. [Compositae] & $\mathrm{H}$ & 0.83 & 0.09 & 0.67 & 1.59 \\
\hline Cymbopogon pendulus (Ness ex Steud.) W.Watson [Gramineae] & $\mathrm{SH}$ & 5.26 & 9.44 & 11.16 & 25.86 \\
\hline Cyanthillium cinereum (L.) H.Rob. & $\mathrm{H}$ & 0.99 & 0.11 & 0.67 & 1.77 \\
\hline Chromolaena odorata (L.) R.M.King \& H.Rob. [Compositae] & SS & 2.08 & 2.02 & 6.03 & 10.13 \\
\hline Crotalaria pallida Aiton [Leguminosae] & $\mathrm{H}$ & 1.77 & 1.27 & 4.46 & 7.50 \\
\hline Clerodendrum infortunatum L. [Labiatae] & SS & 1.15 & 0.77 & 4.24 & 6.18 \\
\hline Cuphea carthagenensis (Jacq.) J.F.Macbr. [Lythraceae] & $\mathrm{H}$ & 1.99 & 0.21 & 0.67 & 2.88 \\
\hline Desmodium triflorum (L.) DC. [Leguminosae] & $\mathrm{H}$ & 4.85 & 6.10 & 7.81 & 18.76 \\
\hline Eleutheranthera ruderalis (Sw. ) Sch.Bip.[Compositae] & $\mathrm{H}$ & 1.97 & 1.99 & 6.25 & 10.21 \\
\hline Hyptis suaveolens (L.) Poit. [Labiatae] & $\mathrm{H}$ & 7.42 & 7.73 & 6.47 & 21.62 \\
\hline Imperata cylindrica (L.) Raeusch. [Gramineae] & $\mathrm{H}$ & 2.97 & 0.21 & 0.45 & 3.65 \\
\hline Ipomoea quamoclit L. [Convolvulaceae] & $\mathrm{C}$ & 1.49 & 0.11 & 0.45 & 2.05 \\
\hline Ichnocarpus frutescens (L.) W.T.Aiton [Apocynaceae] & $\mathrm{C}$ & 1.38 & 0.45 & 2.01 & 3.84 \\
\hline Leucas zeylanica (L.) W.T.Aiton [Labiatae] & $\mathrm{H}$ & 1.24 & 0.09 & 0.45 & 1.78 \\
\hline Mitracarpus hirtus (L.) DC. [Rutaceae] & $\mathrm{H}$ & 30.22 & 43.43 & 8.93 & 82.57 \\
\hline Murraya paniculata (L.) Jack [Rubiaceae] & S & 0.10 & 0.04 & 0.22 & 1.25 \\
\hline Mimosa pudica L. [Leguminosae] & $\mathrm{H}$ & 1.60 & 0.80 & 3.13 & 5.53 \\
\hline Pueraria phaseoloides (Roxb.) Benth & $\mathrm{C}$ & 2.02 & 2.18 & 6.70 & 10.90 \\
\hline Rungia pectinata L. [Acanthaceae] & $\mathrm{H}$ & 2.19 & 0.95 & 2.68 & 5.83 \\
\hline Streptocaulon sylvestre Wight [Apocynaceae] & $\mathrm{SH}$ & 1.18 & 1.02 & 5.36 & 7.56 \\
\hline Sporobolus fertilis (Stued.) Clayton [Gramineae] & $\mathrm{H}$ & 9.93 & 16.41 & 10.27 & 36.62 \\
\hline Setaria viridis (L.) P.Beauv. [Gramineae] & $\mathrm{H}$ & 2.16 & 0.23 & 0.67 & 3.06 \\
\hline Senna occidentalis (L.) Link [Leguminosae] & $\mathrm{H}$ & 1.49 & 0.38 & 1.56 & 3.43 \\
\hline Senna tora (L.) Roxb. [Leguminosae] & $\mathrm{H}$ & 2.41 & 0.52 & 1.34 & 4.26 \\
\hline Triumfetta rhomboidea Jacq. [Malvaceae] & $\mathrm{H}$ & 4.19 & 2.86 & 4.24 & 11.29 \\
\hline Trema orientalis (L.) Blume [Cannabaceae] & $\mathrm{T}$ & 0.10 & 0.11 & 0.67 & 1.77 \\
\hline Wrightia tinctoria R.Br. [Apocynaceae] & $\mathrm{T}$ & 0.50 & 0.02 & 0.22 & 0.74 \\
\hline Zanonia indica L. [Cucurbitaceae] & $\mathrm{C}$ & 0.10 & 0.04 & 0.22 & 1.25 \\
\hline
\end{tabular}


Different indices calculated for the Spring-time vegetation are:

Simpsons Index: $1=0.20$

Menhinick Indices: D $=0.41$

Margalef Indices: R1 $=0.66$

Diversity Indices: The Simpson Index for Concentration of Dominance was observed at 51.78. The value is much higher (0.20) during June, followed by February and April at 0.23 and 0.44 respectively. The Species Richness value was calculated using Menhinick and Margalef indices. Average value of Menhinik Index for Species Richness shows 0.51. During February March it showed a higher value of 0.67, followed by April (0.44) and June (0.41). Margalef index showed the average value of 1.09. February - March study showed much higher value with 1.80 and that was 0.82 in April and 0.66 in June. The species diversity and species richness of this vegetation is moderate with quite high concentration of dominance for the vegetation. This vegetation is quite diverse during post monsoon season. The species richness and concentration of dominance is quite high during April - June.

\section{DISCUSION}

\section{The Flora:}

Phytosociological studies of the SSR in three different seasons has recorded the occurrence of 40 species of angiosperms, representing 18 families within the reserve. It is interesting to note that Monocotyledons are represented by only one family, i.e. Gramineae that too with only (five) species. Of the 18 recorded families, Compositae appeared as the highest represented family with as much as 07 species. It is then followed by Leguminosae (06 spp.), Gramineae (05 spp.), Apocynaceae, Convolvulaceae and Labiatae each with three species, Amaranthaceae

Table 4. Numerical distribution genera and species at the family level in SSR

\begin{tabular}{|c|l|c|c|}
\hline S1. no. & \multicolumn{1}{|c|}{ Family } & $\begin{array}{c}\text { No. of } \\
\text { genera }\end{array}$ & $\begin{array}{c}\text { No. of } \\
\text { species }\end{array}$ \\
\hline $\mathbf{1 .}$ & Amaranthaceae & $\mathbf{1}$ & $\mathbf{2}$ \\
\hline $\mathbf{2 .}$ & Apocynaceae & $\mathbf{3}$ & $\mathbf{3}$ \\
\hline $\mathbf{3 .}$ & Acanthaceae & $\mathbf{1}$ & $\mathbf{1}$ \\
\hline $\mathbf{4 .}$ & Compositae & $\mathbf{7}$ & $\mathbf{7}$ \\
\hline $\mathbf{5 .}$ & Convolvulaceae & $\mathbf{2}$ & $\mathbf{3}$ \\
\hline $\mathbf{6 .}$ & Cucurbitaceae & $\mathbf{1}$ & $\mathbf{1}$ \\
\hline $\mathbf{7 .}$ & Euphorbiaceae & $\mathbf{5}$ & $\mathbf{5}$ \\
\hline $\mathbf{8 .}$ & Gramineae & $\mathbf{5}$ & $\mathbf{6}$ \\
\hline $\mathbf{9 .}$ & Leguminosae & $\mathbf{3}$ & $\mathbf{3}$ \\
\hline $\mathbf{1 0 .}$ & Labiatae & $\mathbf{1}$ & $\mathbf{1}$ \\
\hline $\mathbf{1 1 .}$ & Lauraceae & $\mathbf{1}$ & $\mathbf{1}$ \\
\hline $\mathbf{1 2 .}$ & Lythraceae & $\mathbf{1}$ & $\mathbf{1}$ \\
\hline $\mathbf{1 3 .}$ & Malvaceae & $\mathbf{1}$ & $\mathbf{1}$ \\
\hline $\mathbf{1 4 .}$ & Meliaceae & $\mathbf{1}$ & $\mathbf{1}$ \\
\hline $\mathbf{1 5 .}$ & Rubiaceae & $\mathbf{1}$ & $\mathbf{1}$ \\
\hline $\mathbf{1 6 .}$ & Rutaceae & $\mathbf{1}$ & $\mathbf{1}$ \\
\hline $\mathbf{1 7 .}$ & Ulmaceae & $\mathbf{1}$ & $\mathbf{1}$ \\
\hline $\mathbf{1 8 .}$ & Verbenaceae & $\mathbf{3 7}$ & $\mathbf{4 0}$ \\
\hline & & & \\
\hline
\end{tabular}


with two species and the remaining (Acanthaceae, Cucurbitaceae, Euphorbiaceae, Lauraceae, Lythraceae, Malvaceae, Meliaceae, Rubiaceae, Rutaceae, Ulmaceae, Verbenaceae) 11 families are all represented with just single species (Table 4).

Table 5. Numerical representation of different habit-groups in the SSR

\begin{tabular}{|l|c|c|}
\hline \multicolumn{1}{|c|}{ Habit Group } & \multicolumn{1}{|c|}{$\begin{array}{c}\text { No. of } \\
\text { Species }\end{array}$} & $\mathbf{\%}$ \\
\hline Climber $(\mathrm{C})$ & 6 & 15 \\
\hline Herb $(\mathrm{H})$ & 23 & 57.5 \\
\hline Shrub (S) & 2 & 5 \\
\hline Suffrutescent Herb (SH) & 2 & 5 \\
\hline Suffrutescent Shrub (SS) & 2 & 5 \\
\hline Tree (T) TOTAL & 5 & 12.5 \\
\hline \multicolumn{2}{|c|}{ TO } & \\
\hline
\end{tabular}

The study was conducted mostly during the dry months and the flora was dominated with 23 species $(57.5 \%)$ of herbs, followed by Climbers with six (15\%) species, Trees with five $(12.5 \%)$ species. Shrubs, Suffrutescent Herbs and Suffrutescent shrubs all types are represented by only two (5\% each) species in the habitat. Out of five species of trees, four are represented by seedlings only, i.e. behaving like herbs. Only one stand of Premna mollissima is adult and growing inside the study area (Table 5).

Table 6 shows the five species with higher IVI scores and shows that Cymbopogon pendulus is the most important associated species for S. sylvestre. During February-March when most of the annuals remain in hibernation (in seed condition), some early showers helps $C$. pendulus to sprout with new leaves that was devoid of aerial parts during December - January. Many other plants also try to regenerate during this season including Rungia pectinata, Clerodendrum infortunatum etc.

Table 6. Five species with high IVI scores in the Spring-time (February - March) vegetation [Abbreviations used: as in Table 1]

\begin{tabular}{|l|l|c|c|c|c|}
\hline & \multicolumn{1}{|c|}{ TAXA } & RA & RD & RF & IVI \\
\hline 1. & Cymbopogon pendulus & 13.17 & 42.07 & 20.46 & $\mathbf{7 5 . 7 0}$ \\
\hline 2. & Rungia pectinata & 9.34 & 13.43 & 9.21 & $\mathbf{3 1 . 9 8}$ \\
\hline 3. & Clerodendrum infortunatum & 4.27 & 8.36 & 12.53 & $\mathbf{2 5 . 1 6}$ \\
\hline 4. & Streptocaulon sylvestre & 4.26 & 6.80 & 10.23 & $\mathbf{2 1 . 2 9}$ \\
\hline 5. & Sporobolus fertilis & 5.91 & 7.32 & 7.93 & $\mathbf{2 1 . 1 6}$ \\
\hline
\end{tabular}

Table - 7. Five species with high IVI scores in the Summer-time (April - May) vegetation [Abbreviations used: as in Table 1]

\begin{tabular}{|c|l|c|c|c|c|}
\hline & \multicolumn{1}{|c|}{ Taxa } & RA & RD & RF & IVI \\
\hline 1. & Mitracarpus birtus & 30.23 & 39.90 & 7.77 & $\mathbf{7 7 . 9 0}$ \\
\hline 2. & Chromolaena odorata & 28.87 & 27.94 & 5.70 & $\mathbf{6 2 . 5 1}$ \\
\hline 3. & Hyptis suaveolens & 17.55 & 15.44 & 5.18 & $\mathbf{3 8 . 1 7}$ \\
\hline 4. & Sporobolus fertilis & 4.89 & 6.88 & 8.29 & $\mathbf{2 0 . 0 6}$ \\
\hline 5. & Cymbopogon pendulus & 1.37 & 2.41 & 10.36 & $\mathbf{1 4 . 1 5}$ \\
\hline
\end{tabular}


With increased ambient temperature and precipitation many therophytic plants now produce dense population over the area during April - May (Table 7). Due to their extremely high population structure, Mitracarpus birtus becomes strongly dominating. The situation prevails almost same during June - July (Table 8) rendering Mitracarpus hirtus as the most dominating species.

Table 8. Five species with high IVI scores in the Monsoon-time (June - July) vegetation. [Abbreviations used: as in Table 1]

\begin{tabular}{|l|l|c|c|c|c|}
\hline & \multicolumn{1}{|c|}{ Taxa } & RA & RD & RF & IVI \\
\hline 1. & Mitracarpus birtus & 30.22 & 43.43 & 8.93 & $\mathbf{8 2 . 5 7}$ \\
\hline 2. & Sporobolus fertilis & 9.93 & 16.41 & 10.27 & $\mathbf{3 6 . 6 2}$ \\
\hline 3. & Cymbopogon pendulus & 5.26 & 9.44 & 11.16 & $\mathbf{2 5 . 8 6}$ \\
\hline 4. & Hyptis suaveolens & 7.42 & 7.73 & 6.47 & $\mathbf{2 1 . 6 2}$ \\
\hline 5. & Desmodium triflorum & 4.85 & 6.10 & 7.81 & $\mathbf{1 8 . 7 6}$ \\
\hline
\end{tabular}

However, during all these three study seasons, Cymbopogon pendulus achieved the highest score of IVI. It is also observed within SSR and outside the SSR that S. sylvestre always prefer to remain associated with Cymbopogon pendulus. Its population without this grass do not show good health.

This vegetation is quite diverse during post monsoon season. The species richness and concentration of dominance is quite high during April - June.

However, considering the calculated diversity indices it is realized that the vegetation is highly diverse even though it is dominated by a tall grass, i.e. Cymbopogon pendulus. Another grass Imperata cylindrica also become tall, sometimes over $1 \mathrm{~m}$ and forms very close vegetation. Dicotyledonous plants like Mitracarpus hirtus, Hyptis suaveolens etc. also create very dense vegetation.

\section{Conservation:}

After the rediscovery of Streptocaulon sylvestre in 1990 the natural habitat of this small Critically Endangered angiosperm has been found to be degrading very fast (Das 1996). Quite a few buildings have been constructed on its natural habitat. Only a very small patch has been fenced for its in situ conservation. There is no separate fund for its maintenance. The habitat is maintained like a low herbland during the dry seasons, but turns into a mini-savannah during late and post monsoon seasons.

Previous attempts of the conservation of Streptocaulon sylvestre through germination, transplantation and micropropagation were failed. It was realised that the species should be conserved in situ. Realising this, the "Streptocaulon sylvestre Reserve" has been created within the campus of North Bengal University. But, regular maintenance of the Reserve is very much essential and for with an operation fund need to be generated.

\section{CONCLUSION}

Though herbaceous, the vegetation of "Streptocaulon sylvestre Reserve" is quite dense and the grass population is also extremely high. After fencing the area, it is now protected from grazing and collection of fodder. Previously, this grassland was generally suffering from fire (Intentionally created) during dry season and that was maintaining a lower level of density of 
the vegetation. Because with the increasing density of the vegetation, the amount of light reaching the soil surface considerably. And, Streptocaulon sylvestre is a strictly prostrate plant that generally remain partially covered with grasses.

So, it is essential to reduce the grass cover on the soil regularly during monsoon and in the flowering season of Cymbopogon pendulus i.e. from late September to the end of October. However, all the diversity data express the significance of interference on the natural habitat. Fencing the habitat is also one interference.

It is also interesting to note that the variations within the three sampling season is very less that may be due to the selection of the period that was from a dry and cold season (in February) to only the beginning of the rainy season. Numerous other species of annuals are expected to come up during monsoon and in autumn and early winter a herbaceous temperate vegetation generally develop in the NBU campus. With all those new comers, finally, the plant diversity of this relict vegetation is expected to increase considerably.

The attempts for its ex situ propagation and expansion of the area of occurrence and its domestication as a garden plant also were not positive (Kadir 2001). So, it appears that the conservation of Streptocaulon sylvestre is not an easy job. It should be conserved in in situ condition under strict monitoring.

\section{LITERATURE CITED}

Ahmedullah, M. 2004. Endemism in the Indian Flor. Flora of India ,Introduction Vol . Part II : Botanical Survey of India, Calcutta. Pp. 246 - 265.

Anonymous, 1997. 2015, 2016. Flora of West Bengal, Vols. 1 - 3. Botanical Survey of India, Kolkata.

Awasthi, N. 1992. Addition to the Neogene flora of Kerala coast, India. Geophytology 10: 146 157.

Braun-Blanquet, J. 1932. Plant sociology (Transl. G. D. Fuller and H. S. Conrad). McGraw-Hill, New York.

Champion, H. G. \& Seth, S.K. 1968. A revised survey of the forest types of India. Govt. of India Press, Delhi.

Chatterjee, D. 1939. Floristic Patterns in Indian Vegetation. Proc. Summer School of Botany., Darjeeling, New Delhi.

Chatterjee, D. 1939. Studies on the Endemic Flora of India and Burma Journ. Asiat. Soc. Bengal Sci., 5(1): 19 - 68.

Das, A.P. 1986. On the floristic and palynological surveys of Darjeeling and the adjoining places (Vols. 1\&2). Ph.D. Thesis, University of Calcutta.

Das, A.P. 1995. Diversity of Angiospermic Flora of Darjeeling Hills. In: Pandey, A.K. (ed.), Taxonomy and Biodiversity, New Delhi: CBS Publishers and Distributors, New Delhi. Pp. $118-127$.

Das, A.P. 1996. Rediscovery of Streptocaulon sylvestre wight-an endangered and little known endemic plant of eastern India. J. Bomb. Nat. Hist. Soc. 93(2): 320 - 322.

Das, A.P. 2004. Floristic Studies in Darjeeling Hills. Bull. Bot. Surv. India. 46(1-4): 1 - 18.

Das, A.P. 2013. The present status of the flowering plants of Darjiling and Sikkim. In: Asha Gupta (ed.), Biodiversity Conservation and Utilisation. Pointer Publishers, Jaipur. Pp. 83 - 96.

Das, A.P.; Bhujel, R.B. \& Lama, D. 2008. Plant Resources in the Protected Areas and 
Proposed Corridors of Darjeeling, India. In Biodiversity Conservation in the Kangchanjunga Landscape Eds. N. Chettri, B. Shakya \& E. Sharma. ICIMOD, Kathmandu. Pp. $57-79$

Das, A.P. \& Lahiri, A.K. 1997. Phytosociological studies of the ground covering flora in different types of vegetation in Tiger Hill, Darjeeling district, West Bengal (India). Indian For. 123(12): 1176 1187.

Das, A. P. \& Lungphi, P. 2019. Floristic Studies vis-à-vis Medicinal Plants in the Eastern Himalaya - past and the present status. In: S.K. Tripathi, K. Upadhyaya \& N. Hegde (eds.), Medicinal Plants of India: Conservation and sustainable use. Today \& Tomorrow's Printers and Publishers, New Delhi. Pp. 39 - 56. ISBN: 978817096525

Grierson \& Long 1983 - 2001. Flora of Bhutan including a record of plants from Sikkim and Darjeeling. Volumes 1 \& 2 (in 6 parts). Royal Botanic Garden, Edinburgh.

Hara, H. (ed.) 1966, 1971. The Flora of Eastern Himalaya. $1^{\text {st }} \& 2^{\text {nd }}$ Reports. Tokyo University.

Hara, H.; Chater, A.Q. \& Williams, L.H.J.1982, An Enumeration of the Flowering Plants of Nepal. Vol. III, London.

Hara, H.; Stearn, W.T. \& Williams, L.H.J. 1978. An Enumeration of the Flowering Plants of Nepal. Vol. 1. British Museum (Natural History), London.

Hooker, J.D. 1872 - 1897. The Flora of British India, 7 Vols, L. Reeve \& Co. Ltd., Ashford, Kent. London.

Kadir, A.F.M. Manzur 2001. Ecology of Subbimalayan Herblands in Darjeeling with Special Emphasis on Streptocaulon sylvestre Wight - an Endangered and Endemic Plant. Ph.D. Thesis, North Bengal University, India.

Kadir, A.F.M. Manzur \& Das, A.P. 2007. Reproductive capacity and seedling survivability of Streptocaulon sylvestre Wight - an endangered and endemic plant of Eastern India. Pleione 1(2): $62-68$.

Kadir, AFM Manzur; Ghosh, C. \& Das, A.P. 2009. Interspecific association between Streptocaulon sylvestre Wight and its associated species in sub-himalayan herbland of Darjeeling in India. In: Advances in Plant Biology, ed. S. Mandal \& S. Bhattacharya. Binapani Educational Welfare Trust, Sriniketan, West Bengal. Pp. 195 - 208.

Malhotra, S.K. 1973. Studies on the limest one vegetation of Saha sradhara near Dehra Dunphytosociological studies: Importance Value Index. Indian Flor. 99(2): 102-115

Misra, R, 1968. Ecology Workbook, Oxford \& I.B.H. Calcutta.

Mishra, R. 1971. Phytosociological studies of the crop-weed association of Varanasi. J. Indian Bot. Soc. 50: 142 - 150.

Myers, N. 1988. Threatened Biotas: 'Hotspot' in Tropical Forests. Environmentalist, 8(3): 1 - 20.

Nayar, M.P. 1980. Endemism and patterns of distribution of endemic genera (angiosperms) Journ. Econ. Tax. Bot. 1: 99 - 110.

Nayar, M.P. 1996. Hotspots of Endemic Plants of India, Nepal and Bhutan. Thrivananthapuram, Kerala.

Noltie 1994, 2000. Flra of Bhutan: the Monocotyledons. Vol.3, parts 1 \& 2. Royal Botanic Garden Edinburgh

Ohashi, H., (ed.) (1975): The Flora of Eastern Himalaya. $3^{\text {rd }}$ Report. Tokyo University.

Paczoski, Jozef 1896. Paczoski J. 1896. Przyczynek do historyi badañ flory krajowej. Pamiêtnik fizyograficzny. - Warszawa. - T. XIV. - Dzia ${ }^{3}$ III. - S. 145 - 151. 
70 Phytosociology of 'Streptocaulon sylvestre Reserve'

Phillps 1959. Method of vegetation study. Henry Holt and Co. Inc. New York, U.S.A.

Poore, M.E.D.. 1955. The Use of Phytosociological Methods in Ecological Investigations: II.

Practical Issues Involved in an Attempt to Apply the Braun-Blanquet System; Journal of Ecology 43(1): 245 - 269

Prain, D. 1903. Bengal Plants. Vols. I \&II. West, Newman \& Co., London.

Rai, U. 2006. Characterization of Plant Biodiversity in Darjeeling Hills Using Remote Sensing Techniques. Ph.D. Thesis, University of North Bengal.

Samanta, A.K. 1998. Taxonomical and Phytosociological Studies on the Angiospermic Climbers of Darjeeling and Sikkim Himalayas. Ph.D. Thesis, University of North Bengal, Siliguri.

Sharma, B. 2007. Mapping the Distribution of Some Important Local Tree Species In The Campus of North Bengal University. M. Phil Thesis, University of North Bengal

Shimwell (1971). Description and classification of vegetation. University of Washington Press, Seattle

Sen, K. \& Mandal, R. 2018. Present Status of Terai and Duars Region Biodiversity. International Journal of Science and Research 7(4): 1507 - 1511. ISSN (Online): 2319-7064. DOI: 10.21275/ ART20181812

Tripathi, R.S. \& Mishra, R. 1971. Phytosociological studies of the crop-weed association of Varanasi. J. Indian Bot. Soc. 50: 142-150.

Watson, M.F. 1999. Streptocaulon Wight \& Arn., In: Grierson, A.J.C. \& Long, D.G. (eds.), Flora of Bhutan, Vol. 2, no. 2. Royal Botanic Garden, Edinburgh. Pp. $694-695$.

Wight,R. 1834. Contributions to the Botany of India. Parbury, Allen \& Co., London.

http://www.theplantlist.org

http://www.iucn.com

http://www.nbu.co.org 